\title{
A Dynamical Mean-Field Study of Ferromagnetism in the Periodic Anderson Model
}

\author{
D. Meyer* and W. Nolting \\ Lehrstuhl Festkörpertheorie, Institut für Physik, Humboldt-Universität zu Berlin, Invalidenstr. 110,10115 Berlin
}

(Dated: November 18, 2018)

\begin{abstract}
The ferromagnetic phase diagram of the periodic Anderson model is calculated using dynamical mean-field theory in combination with the modified perturbation theory. Concentrating on the intermediate valence regime, the phase boundaries are established as function of the total electron density, the position of the atomic level and the hybridization strength. The main contribution to the magnetic moment stems from the $f$-electrons. The conduction band polarization is, depending on the system parameters either parallel or antiparallel to the $f$-magnetization. By investigating the densities of states, one observes that the change of sign of the conduction band polarization is closely connected to the hybridization gap, which is only apparent in the case of almost complete polarization of the $f$-electrons. Finite-temperature calculations are also performed, the Curie temperature as function of electron density and $f$-level position are determined. In the intermediate-valence regime, the phase transitions are found to be of second order.
\end{abstract}

\section{INTRODUCTION}

The periodic Anderson model (PAM) represents probably the simplest way of modeling some fifthe rich physics found in Lanthanides and Actinided 1:. B. One believes that most of the physical properties typical for these materials originate from the interplay between the incompletely filled $4 f$ or $5 f$ shells which contain almost localized electrons, and a broad conduction band of $s, p$ and $d$-electrons. In the periodic Anderson model, this is simulated by an atomic-like level with strong on-site Coulomb interaction which hybridizes with an uncorrelated conduction band. Usually the model is further simplified by assuming both the atomic- and conduction states to be non-degenerate. The on-site Coulomb interaction within the $f$-states has to be considered as the largest energy scale of the system since the electrons occupying these states are less screened than the conduction electrons. In the PAM, the Coulomb interaction is further necessary to ensure that the $f$-levels are not fully occupied, thus resembling the situation found in the Lanthanides and Actinides. Due to the incompletely filled $f$-levels, local moments could be formed at every lattice site. It has been a long-standing question whether these moments order magnetically or whether the local moments are screened by conduction band electrons (Kondo screening) 1 . In this paper we will focus on those parameters where the $f$ electron density is non-integer (intermediate valence regime). In this parameter regime, we find a stable ferromagnetic phase and investigate its properties.

Kondo screening has been the subject of extensive investigations act statements 15 . 1 , several approximate or numerical methods have been applied to explore the possibility of ferromagnetism in the PAM, e. g. Hartree-Fack calculations 17, spectrad density approach (SDA) 2.19 , slave-boson techniques2021.22 and dynamic mean-field theory (DMFT) 23. The first methods have severe limitations concerning the description of the low-energy physics. The DMFT-based method promise an improve- ment at this point. However, there are still only few results available on ferromagnetism in the PAM within dynamical mean-field theory.

The antiferromagnetic mhase of the PAM has been discussed in more detail6:20.21,22,23.24. It seems to be widely accepted that close to the symmetric parameter conditions, i. e. half-filling and symmetric DOS, antiferromagnetism has to be expected. This can also be concluded from the following: The PAM can be mapped onto an effective Hubbard model18.19. In the vicinity of the symmetric point the effective Hubbard model will also be close to half-filling. In that case the Hubbard model is-commonly expected to show antiferromagnetic ordering25, therefore the PAM will also have a tendency towards antiferromagnetic ordering as was indeed found]. Ferromagnetism is expected further away from this symmetric point. A ferromagnetic phase was found for the PAM both in the Kondo rogime 6 . 60 . 22 and in the intermediate valence region 18 [19.23. Whereas in the former case, the driving force towards ferromagnetism can be understood via an effective Heisenberg-like coupling of the $f$-spins (RKKY interaction), such a picture is not easily applied to the IV regime due to the non-integer density of $f$-electrons and their effective itineracy.

In this paper, we will investigate ferromagnetism in the PAM using dynamical mean-field theory262728. This theory is based on the assumption of a $\vec{k}$-independent self-energy, which becomes exact in the limit of infinite dimensions $(d=\infty)^{26}$. As pointed out in reference 29, in this limit the lattice coherence and the exchange effects due to the Pauli principle are preserved, contrary to other approaches based on $\frac{1}{N}$ expansion ( $N$ being the degeneracy of the model 30.31 . Especially the exchange effects should be vital for ferromagnetism, therefore the DMFT seems a method of choice.

In the next section, the DMFT together with the modified perturbation theory (MPT) are introduced. The DMFT will lead to a mapping of the PAM onto a singleimpurity model, which then will be solved by applying the MPT. The results concerning zero temperature as 
well as finite temperatures will be presented and discussed in section III.

\section{DMFT AND THE MODIFIED PERTURBATION THEORY}

The periodic Anderson model is defined by its Hamiltonian

$$
\begin{aligned}
H= & \sum_{\vec{k}, \sigma} \epsilon(\vec{k}) s_{\vec{k} \sigma}^{\dagger} s_{\vec{k} \sigma}+\sum_{i, \sigma} e_{f} f_{i \sigma}^{\dagger} f_{i \sigma}+ \\
& V \sum_{i, \sigma}\left(f_{i \sigma}^{\dagger} s_{i \sigma}+s_{i \sigma}^{\dagger} f_{i \sigma}\right)+\frac{1}{2} U \sum_{i, \sigma} n_{i \sigma}^{(f)} n_{i-\sigma}^{(f)}
\end{aligned}
$$

$s_{\vec{k} \sigma}\left(f_{i \sigma}\right)$ and $s_{\vec{k} \sigma}^{\dagger}\left(f_{i \sigma}^{\dagger}\right)$ are the annihilation and creation operators for an electron in a non-degenerate conduction band state (localized $f$-state), and $n_{i \sigma}^{(f)}=f_{i \sigma}^{\dagger} f_{i \sigma}$ is the occupation number operator for the $f$-states. The dispersion $\epsilon(\vec{k})$ describes the propagation of free, i. e. unhybridized conduction electrons, $e_{f}$ is the position of the free $f$-level relative to the center of mass of the conduction band density of states. The hybridization $V$ is taken as a real, $\vec{k}$-independent constant, and finally $U$ is the Coulomb repulsion between two $f$-electrons on the same lattice site.

The quantity of interest will be the $f$-electron Green function

$G_{i i \sigma}^{(f)}(E)=\left\langle\left\langle f_{i \sigma} ; f_{i \sigma}^{\dagger}\right\rangle\right\rangle=\sum_{\vec{k}} \frac{1}{E-\left(e_{f}-\mu\right)-\frac{V^{2}}{E-(\epsilon(\vec{k})-\mu)}-\Sigma_{\vec{k}}}$

To determine this function we employ the dynamical mean-field theory (DMFT) 262 2728. It was shown that in the limit $d \rightarrow \infty$, the self-energy $\Sigma_{\vec{k} \sigma}(E)$ becomes purely local, i. e. $\vec{k}$-independent 26 32. In this case, the self-energy of the PAM is equivalent to the self-energy of a preperly defined single-impurity Anderson model (SIAM)2728 33. The latter has to be defined by the socalled self-consistency condition

$$
\Delta_{\sigma}(E)=E-\left(e_{f}-\mu\right)-\Sigma_{\sigma}(E)-\left(G_{i i \sigma}^{(f)}(E)\right)^{-1}
$$

instead of the usual definition $\Delta=\sum_{\vec{k}} \frac{V^{2}}{E-(\epsilon(\vec{k})-\mu)}$ for the pure SIAM. Using perturbation theory it has been shown in references 34.35 that for a three-dimensional system, the local approximation (equivalent to the limit $d=\infty$ ) provides already for satisfactory results.

Now one is left with the problem to solve the SIAM defined by equation (3). Here we use the modified perturbation theory (MPT) which can be understood as an improvement of the IPT scheme introduced in references 28,36 This method was presented in more detail elsewhere 37, , 8 , so we will restrict ourselves to a short summary here Starting point is the following ansatz for the self-energy 3940 :

$$
\Sigma_{\sigma}(E)=U\left\langle n_{-\sigma}^{(f)}\right\rangle+\frac{\alpha_{\sigma} \Sigma_{\sigma}^{(\mathrm{SOC})}(E)}{1-\beta_{\sigma} \Sigma_{\sigma}^{(\mathrm{SOC})}(E)}
$$

$\alpha_{\sigma}$ and $\beta_{\sigma}$ are introduced as parameters to be determined later. $\Sigma_{\sigma}^{(\mathrm{SOC})}(E)$ is the second-order contribution to nerturbation theory around the Hartree-Fock solution $35,41,42$. The Hartree-Fock solution introduces another free parameter, namely the chemical potential within this calculation: $\tilde{\mu}$. A priori it is not clear whether this should be equal to the chemical potential in the full (DMFT-MPT) calculation, or whether e. g. it should be determined such that the electron density on the impurity site of the SIAM is equal both for the Hartree-Fock and DMFT-MPT calculation. In reference 36 and other papers 1243 yet another condition was used to determine $\tilde{\mu}$. There, the Huttinger theorem 44 or equivalently the Friedel sum rul 45 . 46 was forced to hold. Since these theorems are applicable only for $T=0$, this limits the calculations to zero temperature. In order to access finite temperatures, we used the condition of identical electron densities for the Hartree-Fock and the full calculation $\left(n_{\sigma}^{(f, \mathrm{HF})}=n_{\sigma}^{(f)}\right)$. With this choice, the Friedel sum rule is still fulfilled in a large parameter region as could be shown for the pure SIAM 38 . A more detailed analysis of the different possibilities to determine $\tilde{\mu}$ is found in reference 37 .

Next, the remaining parameters $\alpha_{\sigma}$ and $\beta_{\sigma}$ have to be determined. Instead of using the "atomic" limit of $V=0$ as $E$ vas done e. g. in references 12,36,47 we make use of

$$
\begin{gathered}
M_{\sigma}^{(n)}=\int d E E^{n} A_{\sigma}^{(f)}(E)=\left\langle[\underbrace{\left.\ldots\left[f_{\sigma}, H\right]_{-}, \ldots, H\right]_{-}}_{n \text {-fold commutator }}, f_{\sigma}^{\dagger}]_{+}\right\rangle \\
A_{\sigma}^{(f)}(E)=-\frac{1}{\pi} \Im G_{i i \sigma}^{(f)}\left(E+i 0^{+}\right)
\end{gathered}
$$

where $[A ; B]_{-}\left([A ; B]_{+}\right)$denotes the commutator (anticommutator) of the operators $A$ and $B$ and $\Im x$ denotes the imaginary part of $x$. As indicated, these can be calculated on two different ways, therefore conditions (sum rules) can be derived. To determine $\alpha_{\sigma}$ and $\beta_{\sigma}$, the first four moments $n \in\{0, \ldots 3\}$ have to be used since the $n=0$ - and $n=1$-moments are fulfilled for any $\alpha_{\sigma}$ and $\beta_{\sigma}$. In the $n=3$-moment a higher-order correlation function that we will call bandshift $B_{\sigma}$ is introduced:

$$
\begin{aligned}
& \left\langle n_{\sigma}^{(f)}\right\rangle\left(1-\left\langle n_{\sigma}^{(f)}\right\rangle\right)\left(B_{\sigma}-e_{f}\right)= \\
& \quad=\sum_{k} V_{k \mathrm{~d}}\left\langle s_{k \sigma}^{\dagger} f_{\sigma}\left(2 n_{-\sigma}^{(f)}-1\right)\right\rangle \\
& \quad=-\frac{1}{\pi} \Im \int d E f_{-}(E) \Delta_{\sigma}(E)\left(\frac{2}{U} \Sigma_{\sigma}(E)-1\right) G_{i i \sigma}^{(f)}(E)
\end{aligned}
$$

with the Fermi function $f_{-}(E)=(\exp (\beta E)+1)^{-1}$. 
Now, the solution of the SIAM by the MPT has to be integrated into the DMFT-self-consistency loop28: Starting with a guessed value of $\Sigma_{\sigma}(E)$, equations (2) and (3) are evaluated, and then the new MPT self-energy (4) is calculated for the appropriate SIAM. This procedure is iterated until a self-consistent solution is found. Within this formalism, the spontaneous symmetry-breaking necessary for ferromagnetic solutions can be introduced via spin-asymmetric starting values for the self-consistency cycle. In general, it is not necessary to introduce a symmetry-breaking zero-field.

At this point, let us comment on the quality of our approximative method: Being based on perturbation theory, the MPT is expected to give reliable results for small interaction strengths. For the symmetric SIAM, it was even shown 42 that the perturbative expansion is essentially equivalent to the exact Bethe-ansatz solution 4 , 49 and therefore also valid in the strong coupling regime. For asymmetric parameters, we improve on the perturbation theory by enforcing the correct high-energy expansion for the self-energy and equivalently the Green functions. This is archieved by fulfilling the first four sum rules defined by the spectal moments (5) which leads to the correct determination of the spin-dependent positions and weights of the charge excitations up the order $\frac{1}{U}$ in accordance with reference 5051 . From this, we derive our proposition that the MPT can also be reasonably applied to a PAM in the intermediate-to-strong coupling regime. Although for the high-energy (hightemperature) behaviour this follows from the discussion above, the quality of the special low-energy properties of the PAM within the MPT is not known a priori. To estimate the significance of the results for the intermediate coupling strengths, comparison with exact or numerically exact methods is necessary. For example, one can apply the MPT to a SIAM without the context of the DMFT and compare with the exactly known properties of that modell. This comparison was done in reference 38 and the results can be summarized as follows: The charge excitations are at the proper positions for symmetric as well as asymmetic parameters. Concerning the low-energy behaviour, there is a qualitative agreement but quantitative discrepancies: The Friedel sum rule is fulfilled in a large parameter space off the symmetric point, but the Kondo temperature does not follow an exponential law. A power law is found instead. To summarize, the MPT does qualitatively include essential parts of the Kondo physics, but is prone to deviations concerning energy (temperature) scales. Further tests of the MPT can be done by comparing results for the PAM with different methods based on DMFT, as g. the numerically exact quantum Monte Carlo (QMC)6 6, exact diagonalization (ED)28 or numerical renormalization group theory (NRG) 13 . Comparisons of this kind for the paramagnetic PAM have been published in reference 12 for QMC and reference 52 for ED calculations. Also the results of reference 14 can easily be compared with above-mentioned references. Again, the qualitative features compare well, however temperature scales concerning low-energy properties cannot be reproduced quantitatively. Also, the $V$-dependence seems ${ }^{ \pm}$. be overestimated by the MPT when compared to NRGE3. We do believe that these shortcomings of the MPT do not inhibit the analysis of the ferromagnetic properties of the PAM. In the discussion of our results, we will substanciate this claim.

In this paper, we use the following system parameters: The conduction band is described by a semi-elliptic density of states of unit width centered at $E=0$, thus defining the energy scale. The position of the $f$-level, $e_{f}$ will be given relatively to the center of gravity of the conduction band. The hybridization is taken to be constant. Unless otherwise noted, we chose $V=0.2$. The Coulomb interaction strength $U$ is set to $U=4$ for most calculations, thus representing the largest energy in the system. The total number of electrons $n^{(\text {tot })}, e_{f}$ and the temperature $T$ will be varied. The latter will be given in $K / \mathrm{eV}$ within the energy scale defined by the width of the conduction band.

\section{RESULTS AND DISCUSSION}

Before discussing the results concerning ferromagnetism in the PAM, let us recall some results for the paramagnetic case as they will be important for the following. The density of states (DOS) consists of the two charge excitation peaks approximately at $e_{f}$ and $e_{f}+U$. In addition, a sharp resonance close to the chemical potential is induced by the correlations, the Kondo resonance. Due to lattice coherence, the Kondo resonance is split by the coherence gap in case of symmetric parameters. Another result for the paramagnetic PAM found within the DMFT-MPT scheme is the existence pf local Kondo singlets for large hybridization strengths14. More detailed reviews on the paramagnetic PAM can be found in references 1, 2, 6, 9, $9,14,29,54,55$.

In figure 1, the region of ferromagnetic order is plotted in the $e_{f}$ vs. $n^{\text {(tot) }}$ phase diagram. We have restricted our evaluation to the so-called intermediate valence region (IV region) by positioning the $f$-level within the conduction band which extends from -0.5 to 0.5 . This results in a $f$-occupancy $n^{(f)}$ smaller than unity. Previous investigations have shown fherromagnetism can exist in this parameter regime 18 23.56. The opposite case is the socalled Kondo regime, which is obtained by taking $e_{f}$ well below the lower conduction band edge, and $U$ sufficiently large so that $e_{f}+U$ is clearly above the conduction band. This leads to a nearly half-filled $f$-level. As discussed in the introduction, a half-filled $f$-level should introduce a tendency towards antiferromagnetism which we have not yet investigated. In the following we will therefore concentrate on ferromagnetism in the intermediate valence region, but we will also be noting some trends on how the ferromagnetic phase would continue into the Kondo regime. As can be seen in figure 1, the region of ferromagnetic order is quite large, determined by an upper 
bound for $e_{f}$ and upper and lower bounds for $n^{(\text {tot })}$. As will be shown below, the ferromagnetic region continues into the Kondo regime.

In the left panel of figure 2, the $f-\left(s^{-}\right)$magnetization, $m^{(f, s)}=\frac{n_{\uparrow}^{(f, s)}-n_{\downarrow}^{(f, s)}}{n_{\uparrow}^{(f, s)}+n_{\downarrow}^{(f, s)}}$ is plotted as function of $f$-level position $e_{f}$. The parameters are as in figure 1, the band occupation is given by $n^{\text {(tot) }}=1.3$. Ferromagnetic order breaks down for $e_{f}$ approaching the center of gray ity of the conduction band as it is commonly expected 56 . Within our numerical accuracy, this quantum phase transition is of second order.

In the other limit, i. e. $e_{f}$ below the lower edge of the conduction band $\left(e_{f}<-0.5\right)$, the ferromagnetic solution remains stable. We confirmed that down to $e_{f}=-1.2$ the magnetization behaves as one would extrapolate from figure 2. So, the ferromagnetic phase plotted in figure 1 continues down into the Kondo regime and connects to the ferromagnetic phase found in reference 7. There, the authors found a stable ferromagnetic phase for $n^{\text {(tot) }}<$ 1.6 .

The right picture of figure 2 shows the magnetization as function of the hybridization strength for $e_{f}=-0.4$, the remaining parameters as in the left panel. The ferromagnetic order is destroyed by large hybridizations. This can be understood in terms of local singlet formation. If the conduction electrons screen the magnetic moment

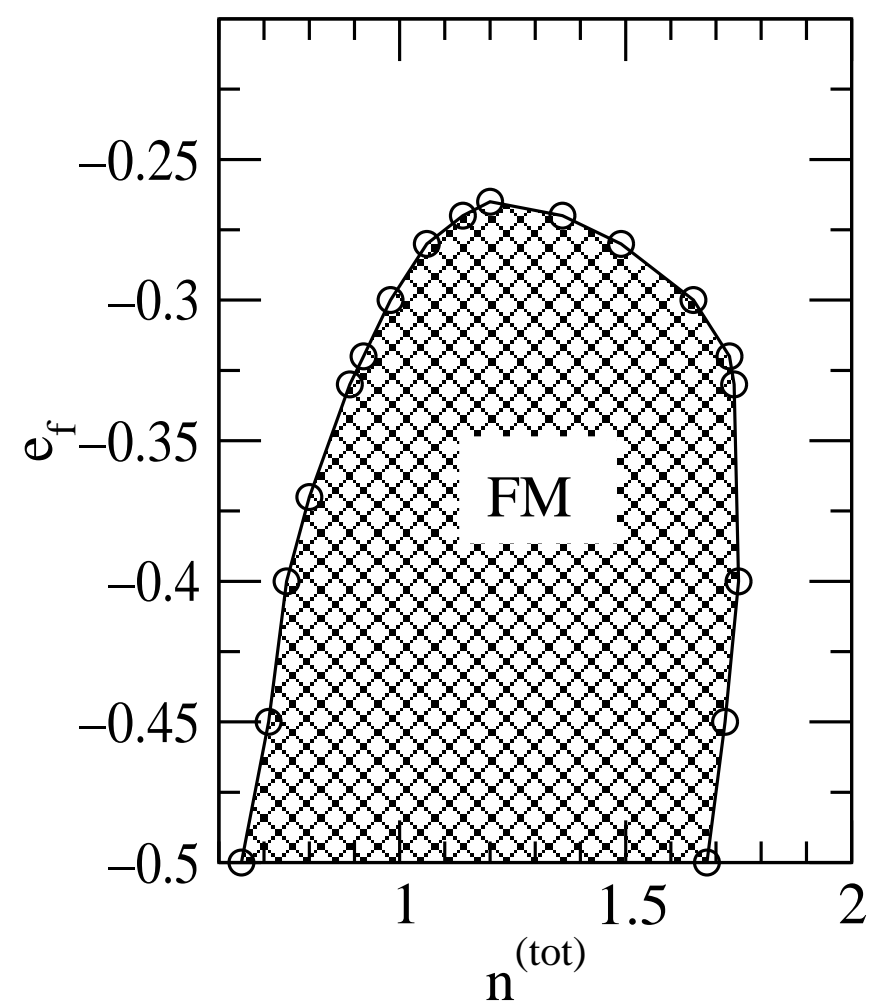

FIG. 1: $e_{f}$ vs. $n^{\text {(tot) }}$ phase diagram for $T=0, U=4$, $V=0.2$ of the localized electrons, as discussed in detail in reference 14, magnetic ordering willnot be possible any more. Comparison with NRG results 13.53 seem to suggest that the $V$-dependence of certain "low-energy" quantities is over-estimated by the MPT, as e. g. the size of the coherence gap. This implies the possibility that the critical $V$ is underestimated since the formation of local Kondo singlets is due to the low-energy physics of the PAM. For $V \rightarrow 0$ the ferromagnetic phase is stable, for numerical reasons no calculations for $V \lesssim 0.03$ have been performed. For $V=0$, the $f$-level and the conduction band decouple completely. The $f$-level basically corresponds to the zero-bandwidth Hubbard model57 in which ferromagnetism is not stable. In figure 2, the thin lines represent the conduction band polarization $m^{(s)}$. In general, it is found to be antiparallel to $m^{(f)}$. However, as will be shown below, a parallel alignment of $m^{(f)}$ and $m^{(s)}$ is also possible for certain parameters.

In figure $\beta$, the $U$-dependence is examined. The inset shows that ferromagnetism sets in at a critical $U_{\mathrm{c}}$ and saturates quickly afterwards. The absolute value of $U_{\mathrm{c}}$ varies strongly with $e_{f}$ : On shifting $e_{f}$ below the conduction band into the Kondo regime, $U_{\mathrm{c}}$ seems to saturate at a value of $U_{\mathrm{c}}^{(\mathrm{KR})} \approx 2.5$. However, there is no true saturation, because when $e_{f}$ is low enough so that the upper charge excitation is close to or even below the chemical potential, the physics of the system will change again. On increasing $e_{f}, U_{\mathrm{c}}$ increases as well and exhibits a sharp upturn at $e_{f} \rightarrow-0.2$. In reference 7 , a ferromagnetic phase was found in the Kondo regime of the PAM for interaction strengths smaller than our $U_{\mathrm{c}}^{(\mathrm{KR})}$. As for the hybridization, the MPT seems to misjudge the energy scale. We will argue below that the Kondo scale is decisive for ferromagnetism in the Kondo regimed, but not in the intermediate valence regime.

In fimro 1 hoth the f-and c-mamnatioation are nlat-

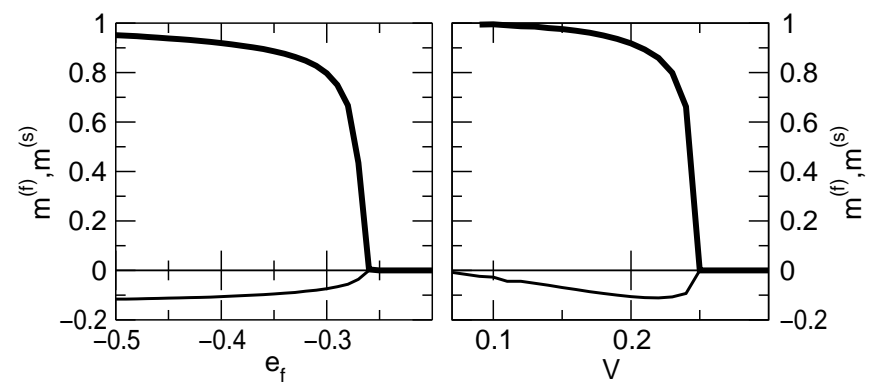

FIG. 2: $f$ - $(s)$-magnetization as function of the hybridization strength $V$ and $f$-level position $e_{f}$ for $T=0, n^{(\text {tot })}=1.3$ and $U=4$ plotted in thick (thin) lines (left panel: $V=0.2$, right panel: $\left.e_{f}=-0.4\right)$. 


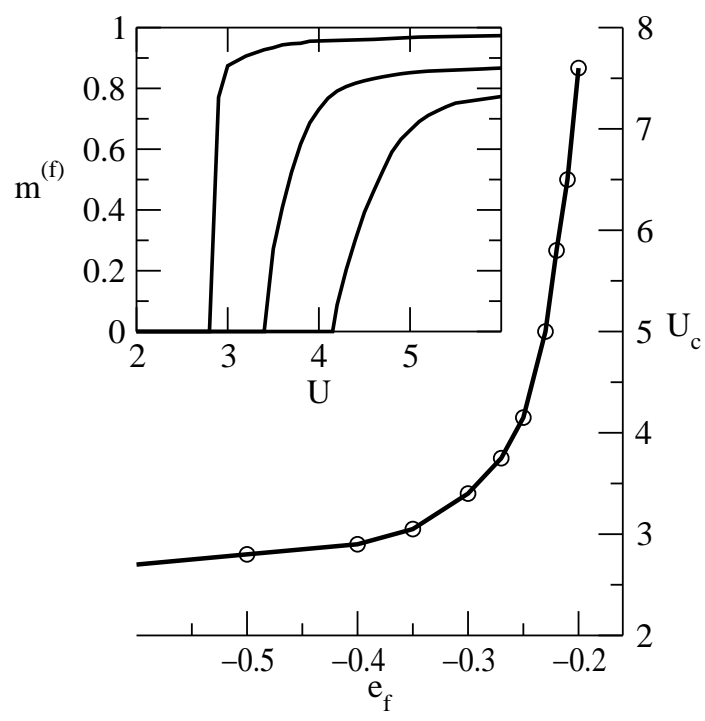

FIG. 3: Critical interaction strength $U_{\mathrm{c}}$ as function of $e_{f}$ for $T=0, V=0.2$ and $n^{\text {(tot) }}=1.3$. The inset shows the respective $f$-magnetization as function of $U$ for $e_{f}=$ $-0.5,-0.3,-0.25$ (from top to bottom).

region depend on $e_{f}$. The lower boundary is in fact determined by the number of $f$-electrons $n^{(f)}$, which itself depends on $e_{f}$ and $n^{\text {(tot) }}$. The phase transition occurs for each $e_{f}$ at that specific electron density, where $n^{(f)}$ drops below $0.56 \pm 0.02$. Approaching the half-filled system, no ferromagnetism is found. This is similar to the findings in reference 7. However as discussed above, antiferromagnetic order is to be expected6.20.22,24 in this case.

For $e_{f}<-0.32$ the ferromagnetic phase can be separated into two regions of different properties. In the low-density regime, the $f$-polarization tends to saturation, the $s$-polarization is positive. On the contrary, in the high-density region $\left(1.1 \lesssim n^{(\text {tot })} \lesssim 1.7\right)$, the $f$ polarization is almost constant, below saturation, and the conduction band polarization is of opposite sign (antiferromagnetic coupling between $f$ - and $s$-band). For more clarity, we will call the first case parallel, the latter case antiparallel coupling of $f$ - and $s$-electrons. This latter case of antiparallel coupling is generally expected for the PAM due to its close relation to the Konde lattice model via the Schrieffer-Wolff transformation 15 . This transformation maps the PAM onto a model with an antiferromagnetic interband-spin exchange. One condition for the Schrieffer-Wolff transformation is that the $f$-level should be half-filled, which is not met here. However, for large total occupation number, $n^{(f)}$ is closer to unity than for small $n^{\text {(tot) }}$. Therefore the finding of parallel coupling for low, and antiparallel coupling for high electron densities is no contradiction to the Schrieffer-Wolff transformation.

In figures 5 and 6, the densities of states are plotted for various band occupations with $e_{f}=-0.4, V=0.2$,

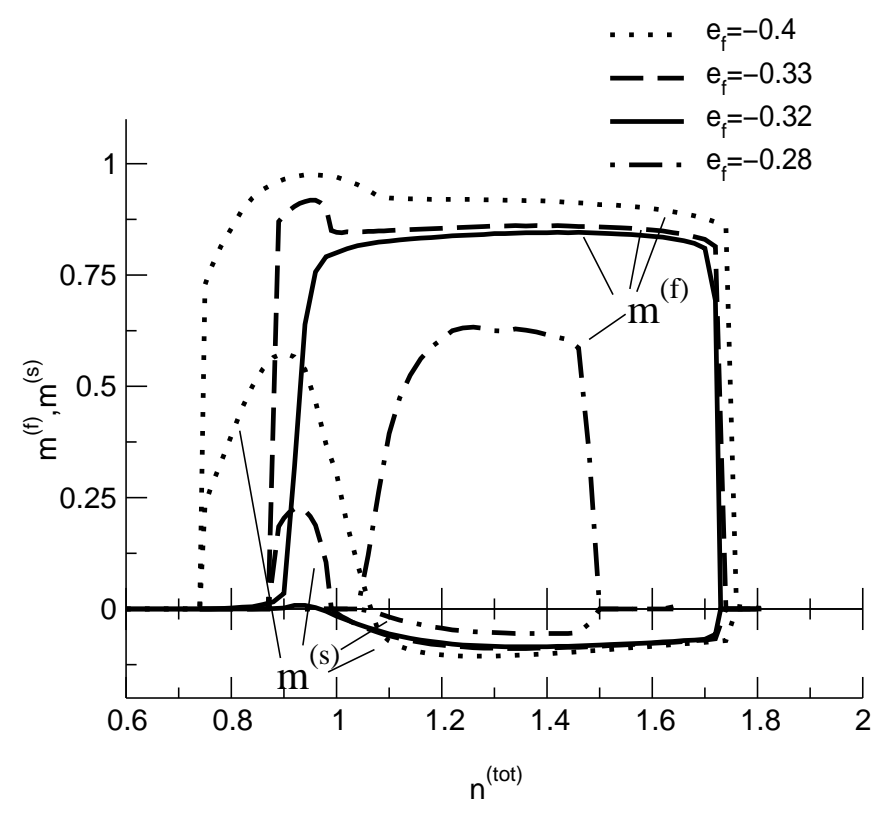

FIG. 4: $f$ - and $s$-magnetization as function of electron density for various $e_{f}$ and $U=4, V=0.2$ and $T=0$.

$U=4$ and zero temperature. The respective left panel corresponds to a projection onto $f$-, the right panel onto $s$-states. The upper charge excitation, located approximately at $E \approx e_{f}+U=3.6$ is not visible in these figures. The arrows indicate the respective position of the chemical potential. To get more insight into the complicated structure of the DOS, we start by explaining figure 5 , where we have forced the system to be paramagnetic. In figure 6, the stable ferromagnetic DOS are shown and will be explained below.

Starting with the $f$-DOS in figure , one observes three structures: the first is the lower charge excitation located roughly at the lower band edge. With increasing electron density, this peak shifts to lower energies. The next structure is the Kondo resonance which is in the vicinity of the chemical potential $\mu$. Due to the asymmetry of the parameters, i. e. the two charge excitation peaks are not symmetric around the chemical potential and the band occupation is well below $n^{(\text {tot })}=2$, the Kondo resonance is not exactly at $E=\mu$ but slightly above. This is in agreement with previous findings 12 . 14 and was also observed in the NBG method 13 and the single-impurity Anderson modell 38 . With increased electron density, the Kondo resonance moves towards higher energies, this is clearly related to the according shift of the chemical potential $\mu$. It is reasonable to expect the Kondo resonance to vanish for decreasing $n^{(f)}$. Within the MPT, the Kondo resonance remains stable for all parameters discussed in this paper. Only for much reduced $n^{(f)}$, where $e_{f}$ is located well above $\mu$, the Kondo resonance disappears. As already mentioned, there is the "coherence gap" in the center of the Kondo resonance for symmetric 


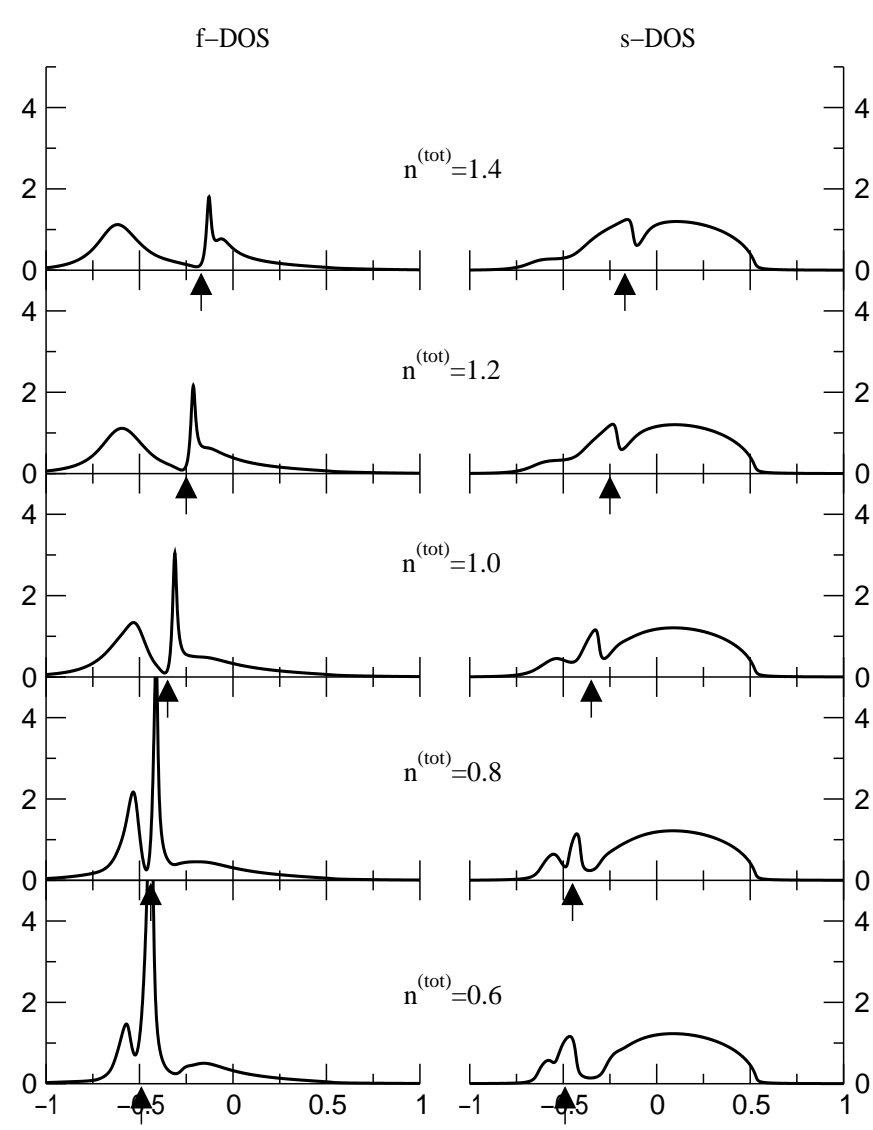

FIG. 5: Paramagnetic $f$ - and $s$-densities of states for $e_{f}=$ $-0.4, V=0.2, U=4$ and $T=0$. The different panels correspond to different electron densities $n^{\text {(tot) }}$

parameters. Due to the asymmetry of the parameters in figure 5 , this gap is closed. Only for the $n^{\text {(tot) }}=1.4$ case (upper panel of figure 5), there is a slight dip in the $f$-DOS visible. This complies with the findings of reference 13. The third feature in the $f$-DOS is a broad structure representing states induced by the hybridization with the conduction band. A fourth feature is of course also present in the DOS, although not plotted in figures 5 and 5 the upper charge excitation which is located roughly at $e_{f}+U=3.6$ and has a FWHM (full width at half maximum) of 0.05 .

All these structures have their counterpart in the conduction-band DOS shown in the right panel of figure 5. The charge excitation induces via the hybridization states in the $s$-DOS. Where the Kondo resonance is visible in the $f$-DOS, the $s$-DOS shows also some anomalies. The original conduction band can also be clearly recognized.

Let us now discuss how the DOS are modified in the ferromagnetic phase. In figure 6 the (stable) ferromagnetic DOS are plotted for the same parameters as in figure 5 . The solid lines represent the spin- $\uparrow$, the dotted lines the spin- $\downarrow$ DOS. For the lowest panel $\left(n^{\text {(tot) }}=0.6\right)$, the system is paramagnetic. For the ferromagnetic so-

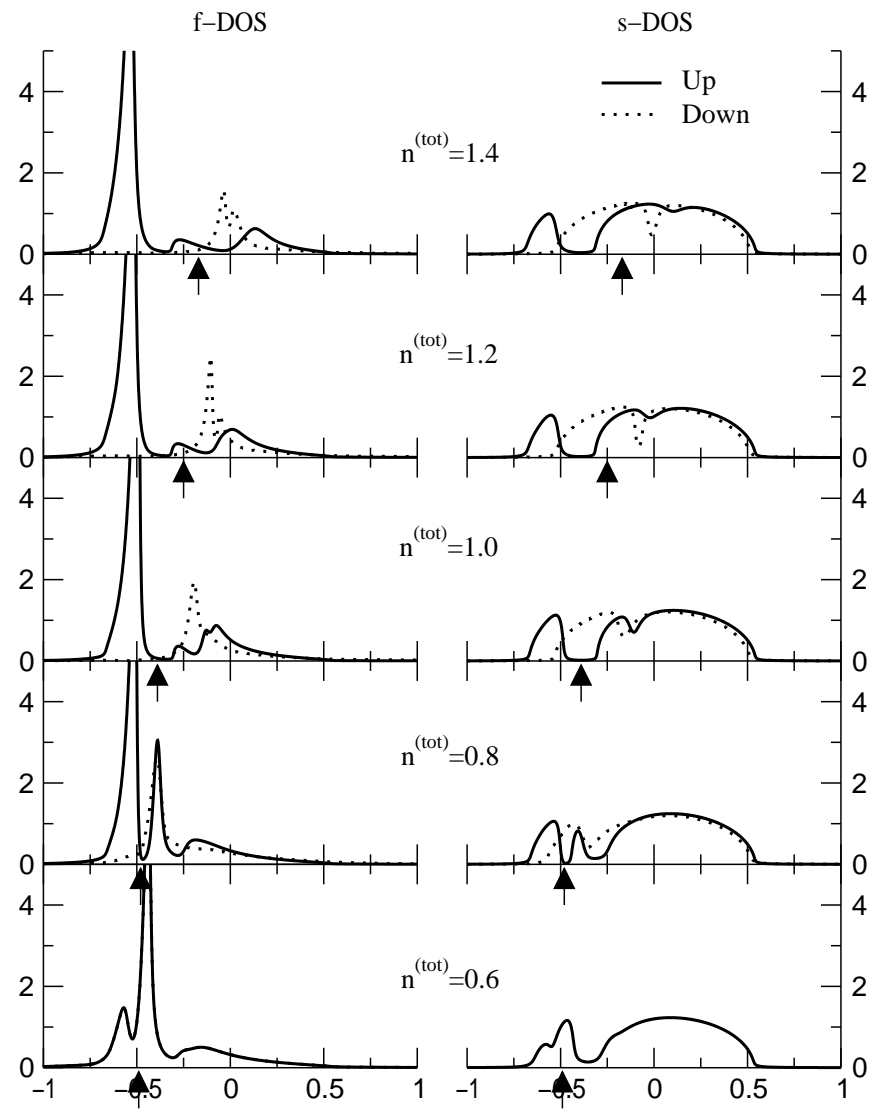

FIG. 6: same as figure 5, but showing the (stable) ferromagnetic solution. The parameters are as in figure 5 , thus corresponding to the dotted line of figure 4. Solid line: spin $\uparrow$; dotted line: spin $\downarrow$.

lutions, the following picture emerges: Firstly, the lower charge excitation is fully polarized, i. e. it consists purely of spin- $\uparrow$ states. Correspondingly, the upper charge excitation, which is not visible, exists only in the spin- $\downarrow$ DOS. Some interesting changes occur to the Kondo resonance: Whereas in the spin- $\uparrow$ channel it disappears almost completely, there is still a peaked structure visible in the spin- $\downarrow$ channel. Contrary to what one would expect for the Kondo resonance, its shift towards higher energies on increasing $n^{\text {(tot) }}$ is stronger than the according shift of $\mu$. It is therefore not located at $\mu$ any more. Although this structure clearly develops from the Kondo resonance, its physical interpretation (Kondo screening) cannot so easily be transfered. Finally there are some substantial changes in the $s$-DOS as well: Due to the full polarization of the lower charge excitation, the hybridizationinduced $s$-states in the same energy range are also purely of majority spin. The feature that corresponds to the Kondo resonance is, for similar reasons, only visible in the spin- $\downarrow-s$-DOS. Finally, in the spin- $\uparrow$ DOS, a new gap appears which separates the lower charge excitation from the remainder of the bare band. Due to this new gap, the system becomes a semi-metal around quarter-filling 
$\left(n^{\text {(tot) }}=1.0\right)$ meaning that the spin- $\uparrow$-DOS vanishes at $\mu$, so only spin- $\downarrow$-electrons contribute to electrical current. The new gap, which we call hybridization gap is not equivalent to the previously discussed coherence gap although the latter is of course also induced by the hybridization. We will explain the differences between these gaps below. This gap is also present in the $f$-DOS, but not visible in the plotted figures because of an artificial broadening of the lower charge excitation which is necessary for numerical reasons.

From these observations the change of sign of the conduction band polarization can be understood: In the case of low electron density, the chemical potential lies within the lower charge excitation. As explained above, due to the hybridization there are only spin- $\uparrow$ conduction band states available in the occupied region of the DOS, the conduction band polarization is necessarily parallel to the $f$-polarization. But on the other hand, with increasing electron density, $\mu$ is increasing as well. The chemical potential now has to cross the hybridization gap which exists only in the spin- $\uparrow$ DOS. As a result, there are more occupied spin- $\downarrow$ than spin- $\uparrow$ states and the conduction band polarization is antiparallel to the $f$-magnetization.

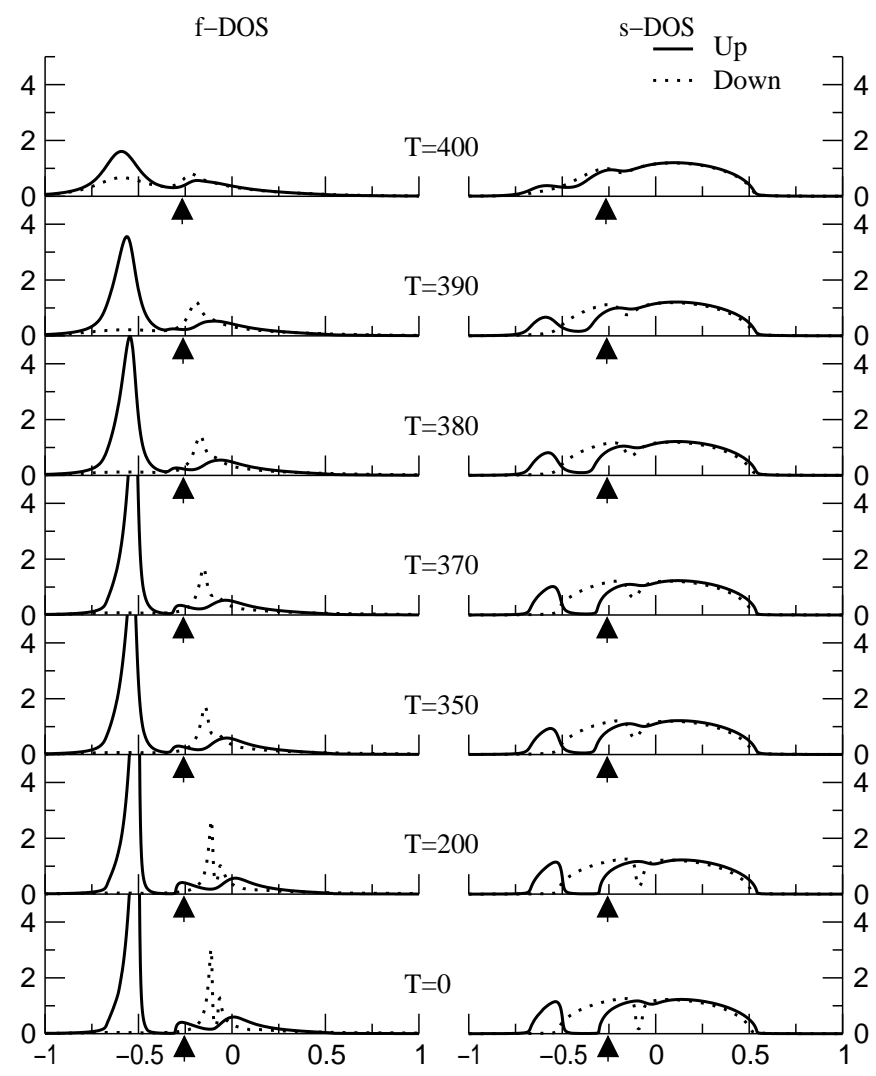

FIG. 7: Temperature dependence of the DOS for $n^{(\text {tot })}=1.2$. The remaining parameters are as in figure 6 .

Let us now discuss the difference between the wellknown coherence gap and the above-described hybridization gap. The coherence gap is found for a symmetric
PAM with on-site hybridization 6 . Contrary to the singleimpurity case, a gap develops in the center of the Kondo resonance located at $E=\mu$. Shifting away from symmetric parameters, this coherence gap gets suppressed, although a dip within the Konderesonance is still visible for a large parameter region 12.13:14. So why is the new gap observed in figure 6 not this coherence gap? This question is answered by the fact that the new gap does not shift with $\mu$ on increasing the electron density. Although there is no proper Kondo resonance in the case of ferromagnetic order, those structures that clearly develop from it do shift with increasing $n^{\text {(tot) }}$. Within this structure, even a dip which is the relic of the coherence gap is visible for $n^{\text {(tot) }}=1.4$. The position of the new gap is neither related to $\mu$ nor the relics of the Kondo resonance, it is rather located at $e_{f}$. And what is the origin of the new gap? Since its position is determined by the value of $e_{f}$ and its width scales with $V$, it reminds of the gap found e. g. within the SDA 18 or alloy analogy 259 , but also for the $U=0$ case of the PAM which is exactly diagonalizablet. This indicates that this gap is due to level-repulsion between the lower $f$-peak and the conduction band, as the coherence gap can be interpreted as level-repulsion between a virtual $f$-level at the fermi energy and the conduction band. To distinguish between these gaps, we call the former "hybridization" and the latter "coherence" gap.

At first sight, it surprises that this gap was not foupd ip any paramagnetic DMFT-based calculation 11.12.13,44.60. This is in our opinion simply due to damping effects caused by the correlation, i. e. a finite imaginary part of the self-energy. In case of saturated ferromagnetism, $\Im \Sigma_{\uparrow}(E)$ vanishes and the corresponding spin- $\uparrow$ DOS should resemble the $U=0$ DOS including the hybridization gap at $e_{f}$. In the spin- $\downarrow$ - or also the paramagnetic DOS, all structures are broadened due to the finite damping. This effectively closes the hybridization gap for these DOS. This proposal can be supported by investigating the temperature-dependence of the DOS. On increasing the temperature, the magnetization will decrease. The system is pushed away from saturation, damping effects occur also for spin- $\uparrow$ electrons. In figure 7, the DOS for $n^{\text {(tot) }}=1.2$ are plotted for various temperatures (the remaining parameters as in figure 6). For these parameters, the Curie temperature is $T_{c} \approx 408$. One can see how the gap closes on approaching $T_{c}$. More complicated is the case of $n^{\text {(tot) }}=0.8$ in figure 6. On the one hand, ferromagnetic saturation is not reached (cf. figure 1) and the hybridization gap is partly closed by damping. And on the other hand, the chemical potential is located in the same energy range, further complicating the situation.

In the following we will discuss the temperature dependence in more detail. Figure 8 shows magnetization curves for $n^{(\text {tot })}=1.2$ and three different values of $e_{f} \in\{-0.5,-0.4,-0.3\}$ (from top to bottom). The lowest line $\left(e_{f}=-0.3\right)$ shows a clear second-order phase transition. The middle case corresponds to the data of figure 7. The phase transition at $T_{c} \approx 408$ is within 


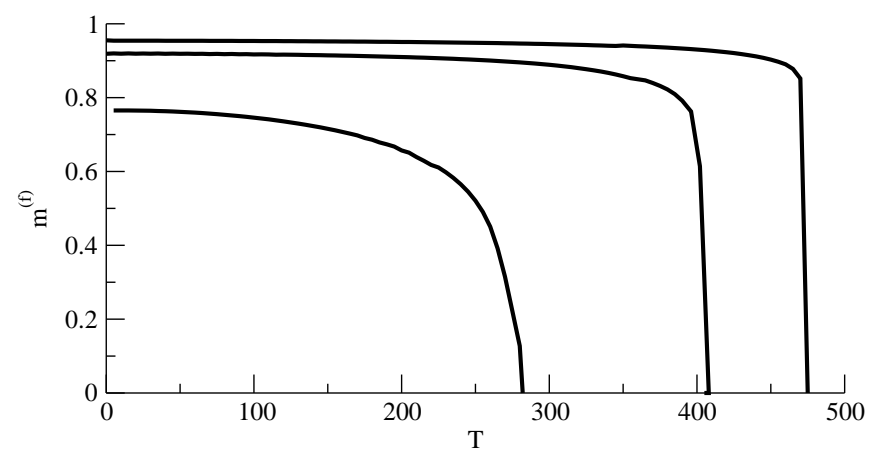

FIG. 8: Magnetization curves for $U=4, V=0.2, n^{\text {(tot) }}=1.2$ and various $e_{f} \in\{-0.5,-0.4,-0.3\}$ (from top to bottom)

numerical accuracy of second order. The demagnetization process is dominated by a transfer of spectral weight from the minority- to the majority-spin spectrum of the lower charge excitation peak as can be seen in figure 7 . However, for the upper charge excitation, plotted in figure 9 for the same parameters as figure 7 , not only the reverse transfer of spectral weight occurs but also a spindependent bandshift can be observed. When decreasing

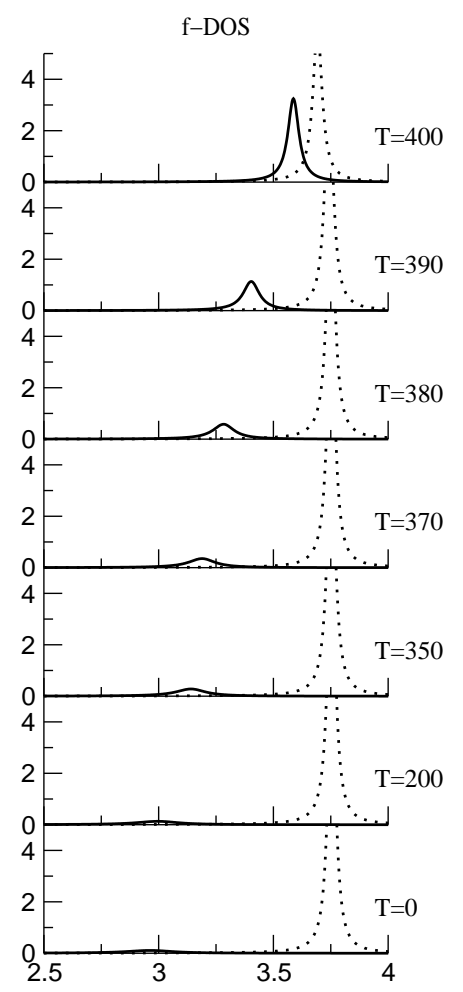

FIG. 9: Upper charge excitation peak of the $f$-DOS for the same parameters as in figure 7

$e_{f}$ towards the lower band edge of the conduction band, the phase transitions become sharper, and finally, first order phase transitions are found for $e_{f} \lesssim-0.5$ within numerical accuracy. This is similar to findings for the
Hubbard mode 1 , where phase transitions were found to be of second order for lower, and of first order for higher electron densities. In the PAM, the density of correlated electrons, $n^{(f)}$, increases on lowering $e_{f}$, therefore the behaviour is analogous to the findings for the Hubbard model. As discussed in reference 51, we cannot exclude that this is an artifact of our method. In figure 10, the

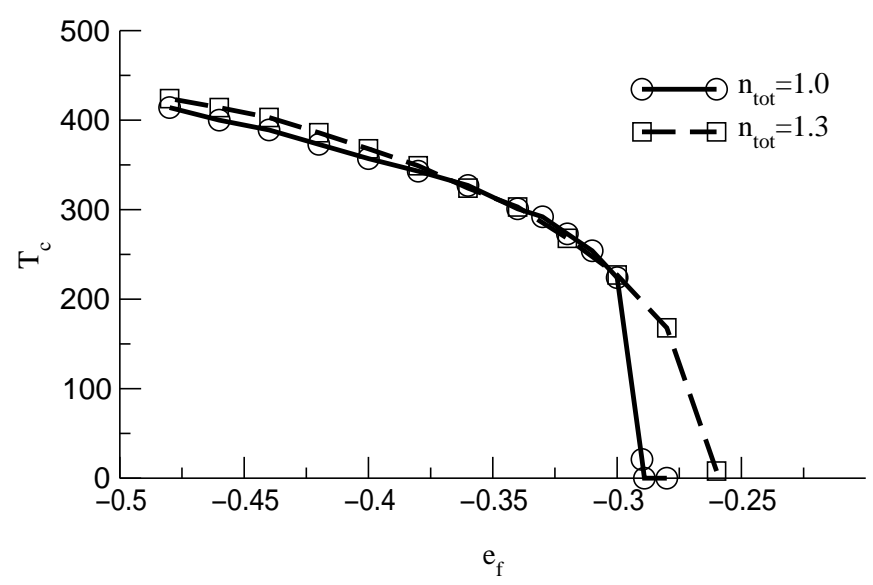

FIG. 10: Curie temperatures as function of $e_{f}$ for $U=4$ and $V=0.2$. The electron density is as indicated.

Curie temperature is plotted as function of the $f$-level position. The trends are very similar to the $T=0$ magnetization as seen in figure 2. For $e_{f}$ approaching the upper critical value, both $T_{c}$ and $m(T=0)$ vanish. In the opposite direction, both quantities keep increasing, however, with diminishing slope. The Curie temperature as function of band occupation is shown in figure 11 for $e_{f}=-0.4$ and $e_{f}=-0.3$.

As already mentioned in the introduction, the magnetism in the Kondo limit of the PAM can be understood in terms of an effective RKKY interaction 61 between the $f$-spins. However, in the intermediate-valence regime under investigation here, the effective RKKY exchange should be suppressed due to the non-integer $f$-electron filling and the effective itineracy of the $f$-electrons as indicated by the broader $f$-peaks in the DOS. We believe that instead of the interband-RKKY exchange, a "narrow-band" mechanism is the main driving force towards ferromagnetism in the IV regime. The effective itineracy of the $f$-electrons due to the hybridization in combination with the strong on-site Coulomb interaction leads to the strong similarities to the well-known Hubbard model62 6364. Recently the existence of ferromagnetism was shown within the Hubbard model 1.65 .66 . One important condition for it is the shape of the free, i. e. $U=0$ DOS entering the model. A highly asymmetric DOS with a divergence or sharp peak close to the band edge enhances the possibility of ferromagnetic order 67. Turning back to the periodic Anderson model we note that this condition favoring ferromagnetic order is excellently met for the hybridization-broadened $f$-level 
in combination with the hybridization-induced $f$-states within the conduction band. Our proposal, that ferromagnetism in the IV regime of the PAM originates from an intraband mechanism is supported by several findings: i) Whereas in the Kondo regime, small values of $U$ can already lead to a ferromagnetic phase, in the IV regime, significantly larger values of $U$ are necessary.

ii) The particular low-energy physics, that are decisive for ferromagnetism in the Kondo regime, have no significant influence on the magnetism in the IV regime.

iii) The critical $n^{\text {(tot)}}$, where ferromagnetism breaks down, is in fact determined by a critical number of correlated $f$-electrons, which is of similar magnitude as for the Hubbard model.

iv) The polarization of the conduction band seems to have almost no influence on the magnetic properties in the IV regime.

Let us discuss these points in more detail: The first point follows directly from figure 3 and reference 7 . In the Kondo regime, where the low-energy physics determine the magnetic propertiest, the MPT probably overestimates the absolute value of $U_{\mathrm{c}}$. However, the sharp increase of $U_{\mathrm{c}}$ for increasing $e_{f}$ indicates the importance of a strong-coupling mechanism in the IV regime. The second point can be made clear by comparing the MPT results with those obtained by two other methods, namely the spectral density approximation (SDA) 18 and the modified alloy analogy (MAA) 59. Whereas all three methods do fulfill the first four moment-sum rules (5) important for the strong-coupling limit, the MPT surely represents a major improvement over the other two methods concerning the low-energy physics. The SDA and the MAA completely neglect the Kondo physics from the very beginning while the MPT includes, as discussed in section II, at least qualitatively the special low-energy properties of the PAM. Now, if the low-energy physics have a major influence on ferromagnetism in the IV regime of the PAM, one would expect a dramatic change of the properties of the ferromagnetic phase. However, in all three methods, the ferromagnetic phase turns out to be very similar, key features as e. g. the phase diagram, but also the unusual conduction band polarization are present in all approximations. This clearly indicates the minor importance of the special low-energy physics of the PAM when describing ferromagnetism in the IV regime. The contrary seems to be the case for the Kondo regime: here the SDA does not give a stable ferromagnetic phase 186 , whereas within the MPT, the ferromagnetic solution remains stable in the Kondo regime and the parameters leading to ferromagnetism agree very well with the results of reference 1 if one ignores the already discussed overestimation of $U_{\mathrm{c}}$. The third point can be clarified by pointing out that for an RKKY mechanism, the density of the conduction band electrons should have a major influence on the effective exchange integrals, and therefore also on phase boundaries. However, as discussed in the context of figure 4 , the lower phase boundary as function of $n^{\text {(tot) }}$ is governed by the number of $f$-electrons and is independent of $n^{(s)}$. This is the expected behaviour for an intraband mechanism as proposed by us. It is clear that for the Kondo regime with fixed $n^{(f)} \approx 1$, the situation is different. Finally let us discuss the influence of the conduction band polarization. It is clear that it is decisive for an RKKY mechanism. However, our results indicate that the magnitude and the unusual behaviour of $m^{(s)}$ have no direct effect on the stability of ferromagnetism in the IV regime (cf. figures 2, 1 and 11). This is a clear indication that an RKKY mechanism is suppressed here.

To summarize the discussion, we believe that two different mechanism are to be considered when discussing ferromagnetism in the PAM: In the Kondo regime, an effective RKKY exchange (interband mechanism) is mainly responsible for ferromagnetism, in the IV regime, the RKKY exchange is suppressed and an intraband mechanism similar to the one found for ferromagnetism in the single-band Hubbard model should be of major importance.

From this proposal it follows that the bandshift (6), which is introduced by the fourth moment of the spectral density, should also be decisive for ferromagnetism in the PAM in the IV regime as it was shown to be in the Hubbard mode 1 1. The low-energy physics, however, are vital in the Kondo regime since the RKKY exchange is believed to origin in the formation of Kondo screening clouds but less important in the IV regime. Therefore the shortcomings of the MPT in reproducing the lowenergy scale of the PAM are nonrelevant for the present investigation.

Of course, both the RKKY and the intraband mechanism are always present, only each respective importance varies. Whereas in the Kondo limit, the effective $f$-electron itineracy becomes negligible, in the IV regime, strong-coupling "Hubbard-like $f$-band" picture is dominant. This explains the smooth transitions found between the to scenarios (cf. figures 2, 3 and 10).

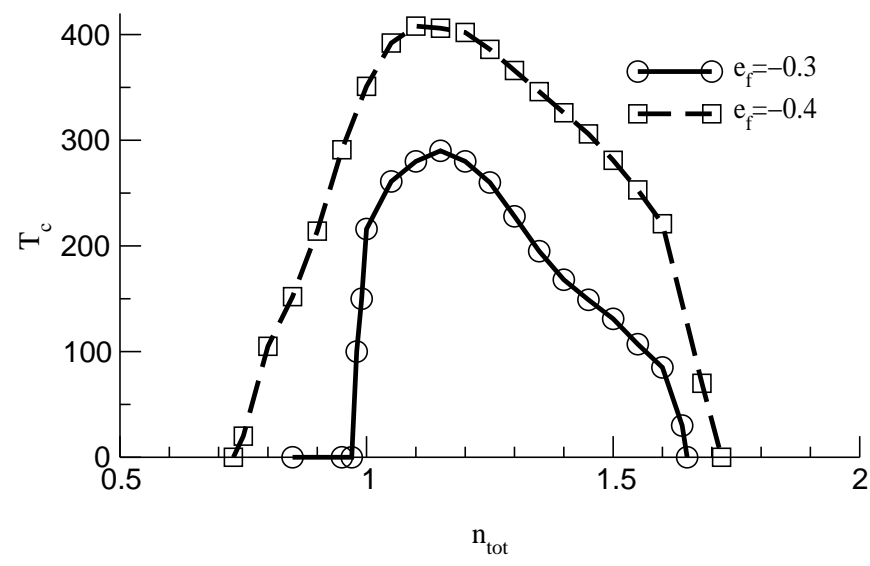

FIG. 11: Curie temperatures as function of electron density for different $e_{f}$. The remaining parameters are as in figure 10 


\section{SUMMARY}

In this paper we have presented a detailed investigation of ferromagnetism in the intermediate valence regime of the periodic Anderson model. We applied the dynamical mean-field theory in combination with the modified perturbation theory. We have shortly discussed the quality of the MPT based on a comparison with exact results for the SIAM and numerically exact results for the paramagnetic PAM. Although the MPT cannot recover the correct exponential energy scale for the low-energy physics, the qualitative features of the low-energy physics emerge properly. And the high-energy features are reproduced with much higher credibility.

We have established the phase diagram as function of the total electron density and the position of the $f$ level. Furthermore, an upper bound of the hybridization strength was found, above which no magnetic ordering seems to be possible due to local Kondo singlet formation 14. The critical interaction strength $U_{\mathrm{c}}$ was found to vary strongly when moving from the intermediate valence to the Kondo regime. The main contribution to the magnetic moment originates from the localized $f$-electrons. However, the conduction band is, due to the hybridization, also polarized. As function of electron density, the conduction band polarization changes sign. For higher electron densities, the conduction-band polarization is antiparallel to the $f$-level magnetization $m^{(f)}$ as one would expect from the Schrieffer-Wolff transfor- mation. For low densities, this transformation is not applicable. Here, the conduction band polarization is parallel to $m^{(f)}$. The densities of states in the ferromagnetic state show rather complicated structures. Besides relics of the Kondo resonance and the dip corresponding to the coherence gap, another gap is present which we named hybridization gap. This gap is closed when approaching the paramagnetic state. Investigating the temperature dependence, we find phase transitions of second and first order. We also have presented the Curie temperatures as function of $f$-level position and total electron density.

Concerning ferromagnetism in the Kondo and Intermediate valence regime, two different pictures emerge: Whereas in the Kondo regime, an Kondo-screening induced RKKY exchange leads to the ordering of the $f-$ spins already for relatively low interactions strengths in the intermediate-valence region, another mechanism becomes more important: due to the effective itineracy of the $f$-electrons, the $f$-levels now represent an strongly correlated narrow band, which by itself can lead to ferromagnetism as known for the Hubbard model.

\section{Acknowledgments}

One of us (D.M.) gratefully acknowledges the support of the Friedrich-Naumann foundation. This work was further supported by the Volkswagen foundation.

$\mathbf{Q}_{*}$

dietrich.meyer@physik.hu-berlin.de

1 A. C. Hewson, The Kondo Problem to Heavy Fermions, Cambridge University Press 1993.

${ }^{2}$ G. Czycholl, Physics Reports 143(5), 2771986.

3 N. Grewe and F. Steglich, Heavy Fermions volume 14 of Handbook on the Physics and Chemistry of Rare Earth page 343, Elsevier Science Publishers 1991.

4 S. Doniach, Physica B 91, 2311977.

5 R. Blankenbecler, J. R. Fulco, W. Gill, and D. J. Scalapino, Phys. Rev. Lett. 58(4), 4111987.

${ }^{6}$ M. Jarrell, Phys. Rev. B 51(12), 74291995.

7 A.N. Tahvildar-Zadeh, M. Jarrell, and J.K. Freericks, Phys. Rev. B 55(6), R3332 1997.

8 P. Nozières, Eur. Phys. J. B 6, 4471998.

9 C. Huscroft, A. K. McMahan, and R. T. Scalettar, Phys. Rev. Lett. 82(11), 23421999.

10 A.N. Tahvildar-Zadeh, M. Jarrell, T. Pruschke, and J.K. Freericks, Phys. Rev. B 60(15), 107821999.

11 T. Pruschke, R. Bulla, and M. Jarrell, Physica B 281-282, 472000.

12 N. S. Vidhyadhiraja, A. N. Tahvildar-Zadeh, M. Jarrell, and H. R. Krishnamurthy, Europhys. Lett. 49(4), 459 2000.

13 T. Pruschke, R. Bulla, and M. Jarrell, Phys. Rev. B 61(19), 127992000.

14 D. Meyer and W. Nolting, Phys. Rev. B 61(20), 13465 2000.

15 H. Tsunetsugu, M. Sigrist, and K. Ueda, Rev. Mod. Phys.

69(3), 8091997

16 T. Yanagisawa and Y. Shimoi, Phys. Rev. B 48(9), 6104 1993.

17 H.J. Leder and B. Mühlschlegel, Z. Phys. B 29, 3411978.

18 D. Meyer, W. Nolting, G.G. Reddy, and A. Ramakanth, phys. stat. sol. (b) 208, 4731998.

19 D. Meyer and W. Nolting, Physica B 259, 9181999.

20 B. Möller and P. Wölfle, Phys. Rev. B 48(14), 103201993.

21 R. Doradziński and J. Spałek, Phys. Rev. B 56(22), R14239 1997.

22 R. Doradziński and J. Spałek, Phys. Rev. B 58(6), 3293 1998.

${ }^{23}$ F. B. Anders, Phys. Rev. Lett. 83(22), 46381999.

24 E. Halvorsen and G. Czycholl, J. Phys.: Condens. Matter 8, 17751996 .

25 P. W. Anderson, In F. Seitz and D. Turnbull, editors, Solid State Physics: Advances in Research and Applications volume 14 page 99. Academic Press New York 1963.

26 W. Metzner and D. Vollhardt, Phys. Rev. Lett. 62, 324 1989.

27 T. Pruschke, M. Jarrell, and J. K. Freericks, Adv. Phys. 44(2), 1871995.

28 A. Georges, G. Kotliar, W. Krauth, and M. J. Rozenberg, Rev. Mod. Phys. 68(1), 131996.

29 F. Gebhard, Phys. Rev. B 44(3), 9921991.

30 P. Coleman, Phys. Rev. B 28, 52551983.

31 N. Read and D. M. Newns, J. Phys. C 16, 32731983.

32 E. Müller-Hartmann, Z. Phys. B 74, 5071989. 
${ }^{33}$ F. J. Ohkawa, Phys. Rev. B 44(13), 68121991.

34 H. Schweizer and G. Czycholl, Solid State Commun. 69(2), 1711989.

35 H. Schweitzer and G. Czycholl, Solid State Commun. 74(8), 7351990.

${ }^{36}$ H. Kajueter and G. Kotliar, Phys. Rev. Lett. 77(1), 131 1996.

37 M. Potthoff, T. Wegner, and W. Nolting, Phys. Rev. B 55(24), 161321997.

${ }^{38}$ D. Meyer, T. Wegner, M. Potthoff, and W. Nolting, Physica B 270, 2251999.

39 A Martin-Rodero, F. Flores, M. Baldo, and R. Pucci, Solid State Commun. 44, 9111982.

40 A Martin-Rodero, E. Louis, F. Flores, and C. Tejedor, Phys. Rev. B 33, 18141986.

41 K. Yamada, Prog. Theo. Phys. 53, 9701975.

42 V. Zlatic̀ and B. Horvatic̀, Phys. Rev. B 28(12), 69041983.

43 O. Takagi and T. Saso, J. Phys. Soc. Japan 68(9), 2894 1999.

44 J. M. Luttinger and J. C. Ward, Phys. Rev. 118(5), 1417 1960.

45 J. Friedel, Can. J. Phys. 34, 11901956.

46 D. Langreth, Phys. Rev. 150(2), 5161966.

47 T. Saso, preprint page to appear in Physica B (SCES99) 1999.

48 N. Andrei, Phys. Rev. Lett. 45(5), 3791980.

49 A. M. Tsvelick and P. B. Wiegmann, Adv. Phys. 32(4), 4531983.

50 A. Harris and R. Lange, Phys. Rev. 157(2), 2951967.

51 M. Potthoff, T. Herrmann, T. Wegner, and W. Nolting, phys. stat. sol. (b) 210, 1991998.
52 T. Saso, J. Phys. Soc. Japan 66(4), 11751997.

53 T. Pruschke, private communication.

${ }^{54}$ H. Schweizer and G. Czycholl, Z. Phys. B 74, 3031989.

55 H. Schweitzer and G. Czycholl, Z. Phys. B 79, 3771990.

56 T. M. Rice and K. Ueda, Phys. Rev. Lett. 55(9), 9951985.

57 W. Nolting, Viel-Teilchen-Theorie volume 7 of Grundkurs: Theoretische Physik, Friedr. Vieweg \& Sohn Verlagsgesellschft mbH Braunschweig/Wiesbaden 3 edition 1997.

58 J. R. Schrieffer and P. A. Wolff, Phys. Rev. 149(2), 491 1966.

59 G. G. Reddy, D. Meyer, S. Schwieger, A. Ramakanth, and W. Nolting, submitted.

60 A.N. Tahvildar-Zadeh, M. Jarrell, and J.K. Freericks, Phys. Rev. Lett. 80(23), 51681998.

61 W. Nolting, S. Rex, and S. Mathi Jaya, J. Phys.: Condens. Matter 9, 13011997.

62 J. Hubbard, Proc. R. Soc. London, Ser. A 276, 2381963.

63 M. C. Gutzwiller, Phys. Rev. Lett. 10(5), 1591963.

64 J. Kanamori, Prog. Theor. Phys 30, 2751963.

65 M. Ulmke, Eur. Phys. J. B 1, 3011998.

66 T. Obermeier, T. Pruschke, and J. Keller, Phys. Rev. B 56(4), R8479 1997.

67 D. Vollhardt, N. Blümer, K. Held, M. Kollar, J. Schlipf, and M. Ulmke, Z. Phys. B 103, 2831997.

68 D. Meyer, Diploma thesis Humboldt-Universität zu Berlin May 1997. (http://tfk.physik.hu-berlin.de/ dmeyer). Although a ferromagnetic solution can be found within the SDA for $e_{f}$ below the conduction band, its free energy is then much larger than the one of the paramagnetic solution. 